\title{
A CHARACTERIZATION OF NONCHAOTIC CONTINUOUS MAPS OF THE INTERVAL STABLE UNDER SMALL PERTURBATIONS
}

\author{
D. PREISS AND J. SMÍTAL
}

\begin{abstract}
Recent results of the second author show that every continuous map of the interval to itself either has every trajectory approximable by cycles (sometimes this is possible even in the case when the trajectory is not asymptotically periodic) or is $\varepsilon$-chaotic for some $\varepsilon>0$. In certain cases, the first property is stable under small perturbations. This means that a perturbed map can be chaotic but the chaos must be small whenever the perturbation is small. In other words, there are nonchaotic maps without "chaos explosions". In the paper we give a characterization of these maps along with some consequences. Using the main result it is possible to prove that generically the nonchaotic maps are stable.
\end{abstract}

\section{INTRODUCTION}

Let $\mathscr{C}(I, I)$ denote the class of continuous maps $I \rightarrow I$, where $I$ is a compact real interval. The trajectory of $x \in I$ with respect to $f$ is the sequence $\left\{f^{n}(x)\right\}_{n=0}^{\infty}$ where $f^{n}$ denotes the $n$th iterate of $f$; the $\omega$-limit set of this trajectory is denoted by $\omega_{f}(x)$. By $\operatorname{Per}(f)$ we denote the set of periodic points of $f$; the period of a point is always its smallest positive period. The metric in $\mathscr{C}(I, I)$ is the uniform one and will be denoted using $\|\cdot\|$.

Asymptotic properties of trajectories have been intensively considered since the middle of the sixties when excellent papers by A. N. Śarkovskii appeared. The main reason is applicability of these results in mathematical modelling. Important are maps with trajectories having regular asymptotical behavior, like asymptotical periodicity. However, this property can be easily destroyed by arbitrarily small perturbations of the maps and the corresponding unstable models. In connection with this there appeared some results guaranteeing the stability. In [15] it is shown that every map $f$ having no periodic orbits of period different from $1,2, \ldots, 2^{n}$, for some $n$, with $\operatorname{Per}(f)$ nowhere dense, is stable. This result was generalized [16] to the maps $f$ with $\operatorname{Per}(f)$ nowhere dense, which have all trajectories asymptotically periodic. These maps can have cycles of period $2^{n}$ for every $n$, but no other cycles, and are characterized e.g. by the property that $\operatorname{Per}(f)$ is closed [12] (cf. also [10]).

Received by the editors January 22, 1988.

1980 Mathematics Subject Classification (1985 Revision). Primary 58F08, 58F10, 54H20, 26 A18. 
In [14 and 7] the following classification of mappings is given, generalizing results from [8]: Every $f \in \mathscr{C}(I, I)$ has exactly one of the following properties:

A. Every trajectory of $f$ is approximable by cycles. In other words, for any $x$ and any $\varepsilon>0$ there is $p \in \operatorname{Per}(f)$ such that

$$
\limsup _{n \rightarrow \infty}\left|f^{n}(x)-f^{n}(p)\right|<\varepsilon .
$$

In application, such trajectories are not distinguishable from asymptotically periodic ones.

B. For some $\varepsilon>0, f$ has a nonempty perfect $\varepsilon$-scrambled set $S$, i.e., such a set that for any $x, y \in S, x \neq y$, and any $p \in \operatorname{Per}(f)$,

$$
\begin{aligned}
& \limsup _{n \rightarrow \infty}\left|f^{n}(x)-f^{n}(y)\right|>\varepsilon, \\
& \liminf _{n \rightarrow \infty}\left|f^{n}(x)-f^{n}(y)\right|=0, \\
& \limsup _{n \rightarrow \infty}\left|f^{n}(x)-f^{n}(p)\right|>\varepsilon .
\end{aligned}
$$

Every map having property $B$, is called $\varepsilon$-chaotic. It turns out that the $\varepsilon$ chaoticity is a weaker property than positive topological entropy (which is sometimes considered as the criterium of chaos). Actually, for any $f \in \mathscr{C}(I, I)$ with zero topological entropy (denoted by $h(f)=0$ ) each of the following, mutually excluding types of behavior, is possible [14]:

1. Every trajectory of $f$ is asymptotically periodic (or equivalently, for any $x, \omega_{f}(x)$ is a cycle).

2. There is some $x$ with $\omega_{f}(x)$ infinite, but $f$ is not chaotic.

3. $f$ is $\varepsilon$-chaotic for some $\varepsilon>0$.

In this paper we give a full characterization of the nonchaotic maps (i.e., maps of the first two types) that are stable under small perturbations in the sense that the perturbed map can be chaotic but the chaos must be small. (In other words, no "chaos explosions" are possible for such maps; Theorem 2.2 below). As a consequence, we obtain another useful result. It turns out that the stability is a generic property of nonchaotic maps (Remark 2.9 below).

The paper is organized as follows. The Main Result with some consequences and remarks are in $\S 2$. Next $\S 3$ contains some preliminary results. Nevertheless, Lemma 3.7 and the ideas surrounding it have particular relevance beyond the proof of Theorem 2.2 which is given in $\S 4$.

\section{MAin Results}

We begin with

2.1 Definition. A nonchaotic map $f \in \mathscr{C}(I, I)$ is called stable if for any $\varepsilon>0$, any $g \in \mathscr{C}(I, I)$ sufficiently near to $f$ (relative to the uniform metric) has every trajectory $\varepsilon$-approximable by cycles (i.e., if for any $x$ there is a $p \in \operatorname{Per}(g)$ such that $(1.1)$ is true for $f$ replaced by $g$ ). 
2.2 Theorem. A nonchaotic map $f \in \mathscr{C}(I, I)$ is stable if and only if the following two conditions are satisfied:

(i) $\operatorname{Per}(f)$ is nowhere dense (or, equivalently, $\operatorname{Per}(f)$ is a first category set, or even, $\operatorname{Per}(f)$ contains no interval $)$;

(ii) for any infinite $\omega$-limit set $\omega_{f}(x)$ and any positive integer $n$ there is a system $\mathscr{I}_{n}=\left\{I_{n}^{i} ; 1 \leq i \leq 2^{n}\right\}$ of periodic intervals such that

$$
\omega_{f}(x)=\bigcap_{n=1}^{\infty}\left(\bigcup \mathscr{I}_{n}\right)
$$

2.3 Remark. If $h(f)=0$ (in particular, if $f$ is nonchaotic) then for any infinite $\omega$-limit set $\omega_{f}(x)$ and for any $n$, there is always a system $\mathscr{I}_{n}$ of $2^{n}$ closed periodic intervals of period $2^{n}$ covering $\omega_{f}(x)$ (cf. [14]) but (2.1) may be false even in the case when every $\mathscr{I}_{n}$ is a minimal periodic cover of period $2^{n}$.

In this case, however, $\bigcap_{n=1}^{\infty}\left(\cup \mathscr{I}_{n}\right)$ has a nonempty interior (see, e.g., proof of Proposition 4.4 below) and when $J$ is any interval contained in it then $f^{k}(J) \cap J=\varnothing$ for every $k$, since the sets $J, f(J), \ldots, f^{k}(J)$ lie in disjoint components of the set $\bigcup \mathscr{I}_{n}$ for every sufficiently large $n$. Thus we have the following

2.4 Corollary. Every nonstable map $f \in \mathscr{C}(I, I)$, that is not chaotic, has a wandering interval.

Note that also every chaotic map with zero topological entropy must have wandering intervals (cf. [14]).

2.5 Corollary. If $f \in \mathscr{C}^{\infty}(I, I)$ is unimodal with $h(f)=0$ and with a nonflat critical point (i.e., certain derivative of $f$ is nonzero at the critical point) then $f$ is nonchaotic and stable.

This easily follows from Corollary 2.4 since the described map has no wandering intervals (cf. [5]).

2.6 Remark. The converse statement to Corollary 2.4 is not true. It is easy to find a map $f \in \mathscr{C}(I, I)$ having only one periodic point-a fixed point $a$, with wandering intervals converging to it. But the converse is false also in the case when $f$ has a wandering interval converging to an infinite $\omega$-limit set (cf. Example 5.6 in [14]).

2.7 Remark. The technique of blowing up orbits (cf., e.g., [6]) can be used to create chaotic as well as nonchaotic nonstable maps. Let $f:[0,1] \rightarrow[0,1]$ be a unimodal map with $h(f)=0$, having a cycle of period $2^{n}$, for every $n$. Then $f$ has a unique $\omega$-limit set-a Cantor-type set $\omega_{f}(x)$ (cf., e.g., [4]). Now take an arbitrary $a \in \omega_{f}(x)$ and blow up its backward orbit $B(a)=\left\{y ; f^{i}(y)=a\right.$ for some $i \geq 0\}$. In other words, replace every point in $B(a)$ by a suitable interval and compress the rest of the points in such a way that the resulting 
map $\tilde{f}$ again belongs to $\mathscr{C}(I, I)$ with $I=[0,1]$. It is known [4] that $B(a)$ contains points interior relative to $\omega_{f}(x)$. Let $z$ be such a point. Blowing up $z$ gives an interval with end-points $u<v$, which clearly belong to $\omega_{\tilde{f}}(x)$. It needs some considerations to show that $u$ and $v$ cannot be separated by disjoint periodic intervals of $\tilde{f}$. But this is equivalent to the condition that $\tilde{f}$ is $\varepsilon$-chaotic with $\varepsilon$ arbitrarily close to $v-u$ (see [14] for details). Note that this construction cannot give maps $g$ with $h(g)=0$ having infinite attractors with isolated points (cf. [3]) which are chaotic, too [14].

If, on the other hand, $f:[0,1] \rightarrow[0,1]$ is a nonchaotic map possessing an infinite $\omega$-limit set $\omega_{f}(x)$, and if for some $b \in \omega_{f}(x)$, the backward orbit $B(b)$ (which is always dense in $\omega_{f}(x)$ ) contains no points interior relative to $\omega_{f}(x)$, then blowing up $B(b)$ gives a nonchaotic map $\tilde{f}$ (the property, that every two points $u \neq v$ belonging to an infinite $\omega$-limit set of $f$ can be separated by periodic intervals, remains unchanged). But $\omega_{\tilde{f}}(x)$ does not satisfy condition (ii) from Theorem 2.2 and consequently, $\tilde{f}$ is nonstable. An example of $f$ with this property is the map $g$ from the proof of Theorem 2.7(ii) in [14]: then $\omega_{f}(x)$ is the Cantor middle-third set and we can take, e.g., $b=1$.

2.8 Remark. Any map $f \in \mathscr{C}([0,1],[0,1])$, nondecreasing in $[0, a]$, nonincreasing in $[b, 1]$ and constant in $[a, b]$ with $a<b$, which has periodic points of period $2^{n}$ for every $n$, is chaotic [9]. Such a map can be clearly made to be from $\mathscr{C}^{\infty}$.

However, when $f$ is unimodal and sufficiently smooth, and if $h(f)=0$, then we believe that $f$ is nonchaotic and stable. The proof of this statement remains an open problem.

2.9 Remark. Let $f \in \mathscr{C}(I, I)$ be nonchaotic. Then the $\omega$-limit sets of $g=f^{m}$ where $m=2^{n}$, have small diameters if $n$ is sufficiently large. This follows, e.g., from [14]. Using this it is possible to show that the class of stable nonchaotic maps is dense in the class of nonchaotic maps from $\mathscr{C}(I, I)$. Theorem 2.2 then implies that, for a given $\varepsilon>0$, the class $A_{\varepsilon} \subset \mathscr{C}(I, I)$ of maps having all trajectories $\varepsilon$-approximable by cycles, contains an open and dense subset of the set of nonchaotic maps. Consequently, the nonchaotic maps are generically stable.

\section{Preliminary Results}

First we recall certain known results that will be useful in the sequel.

3.1 Proposition (A. N. Šarkovskii). Let $\varphi \in \mathscr{C}(I, I)$.

(i) For any $u \in \overline{\operatorname{Per}(\varphi)} \backslash \operatorname{Per}(\varphi)$ there is a $v \in I$ such that $\omega_{\varphi}(v)$ is infinite and contains $u$.

(ii) Let $\varphi$ have no cycle of period $\neq 2^{n}$ and let $\Omega(\varphi)$ be the set of nonwandering points of $\varphi$ (i.e., $u \in \Omega(\varphi)$ means that for every neighborhood $U$ 
of $u$ there is some $n>0$ with $\left.\varphi^{n}(U) \cap U \neq \varnothing\right)$. Then $\Omega(\varphi)=\overline{\operatorname{Per}(\varphi)} \cup A$ where $A$ is a (countable) set consisting of points isolated in $\Omega(\varphi)$.

Proof. For (i) see [13], (ii) was proved in [11].

3.2 Remark. For any $\varphi \in \mathscr{C}(I, I)$, the following conditions are equivalent.

(i) $h(\varphi)>0$;

(ii) $\varphi$ has a cycle of period not a power of 2 ;

(iii) $\varphi$ has a horseshoe, i.e., there are closed intervals $J_{0}, J_{1} \subset I$ with at most one point in common, and an integer $n>0$ such that

$$
\varphi^{n}\left(J_{0}\right) \cap \varphi^{n}\left(J_{1}\right) \supset J_{0} \cup J_{1} \text {. }
$$

The equivalence has been proved by Šarkovskii and Misiurewicz and complete references can be found, e.g., in [14].

3.3 Proposition. Let $\varphi \in \mathscr{C}(I, I)$ and let $J_{0}, J_{1}$ be disjoint closed intervals satisfying (3.1). Then $\varphi$ is $\varepsilon$-chaotic with $\varepsilon=\frac{1}{2} \operatorname{dist}\left\{J_{0}, J_{1}\right\}$.

Proof. The result is implicitly contained in the proof of Theorem 1 in [7].

Next we prove some lemmas that will simplify the proof of Theorem 2.2.

3.4 Lemma. Let $\varphi \in \mathscr{C}(I, I), h(\varphi)=0$, let $\varphi^{m}(p)=p$ for some $p \in \operatorname{Per}(\varphi)$ and let $m=2^{k}$. Set $\psi=\varphi^{2 m}$. Then there are no two points $x, y \in I$ with

$$
\psi(y) \leq x<p<y \leq \psi(x) .
$$

Proof. Assume there are $x, y$ satisfying (3.2). First we find a point $q$ such that

$$
\psi(q)<p<q=\psi^{2}(q) .
$$

If $\psi(y)=x$ and $y=\psi(x)$, then $q=y$ has the required properties. If $\psi(y)<x$ or $y<\psi(x)$ then there is the smallest $z \in(p, y]$ with $\psi(z)=x$. It is easy to see that $\psi^{2}(z)>z$, and since $\psi^{2}(b) \leq b$ for $b$ the right-hand end-point of $I$, there is the least $q>z$ with $\psi^{2}(q)=q$.

Now for $v \in(z, q), \psi(v) \neq p$. To see it assume the contrary. Then $\psi^{2}(v)<v$ and since $\psi^{2}(z)>z$, there would be $\psi^{2}(w)=w$ for some $w \in$ $(z, v) \subset(z, q)-\mathrm{a}$ contraction. Consequently, $\psi(q) \leq p$ and since $\psi(q)=p$ would imply $\psi^{2}(q) \neq q$, we have proved (3.3).

Finally, by (3.3), $q$ is a periodic point of $\varphi^{m}$ of order 4. Since $h\left(\varphi^{m}\right)=0$, the even iterates of $q$ must be separated by the fixed point $p$ from the odd ones (cf. [2]), i.e., $\varphi^{m}(q), \varphi^{3 m}(q)<p<q, \varphi^{2 m}(q)$. But in our case, $\varphi^{2 m}(q)=$ $\psi(q)<q-$ a contraction.

3.5 Lemma. Let $\varphi \in \mathscr{C}(I, I)$ with $h(\varphi)=0$, and let $\varphi(y) \leq x<y \leq \varphi(x)$, for some $x, y$. Then there is not fixed point of $\varphi$ in $[\varphi(y), x] \cup[y, \varphi(x)]$.

Proof. Denote by $p$ a fixed point of $\varphi$ lying between $x$ and $y$, and assume that e.g. $\varphi$ has a fixed point $q \in[y, \varphi(x)]$. Set $J_{0}=[x, p], J_{1}=[y, q]$. Then 
$\varphi\left(J_{1}\right) \supset[\varphi(y), q] \supset J_{0} \cup J_{1}$ and $\varphi\left(J_{0}\right) \supset[p, \varphi(x)] \supset J_{1}$, so $\varphi^{2}\left(J_{0}\right) \supset J_{0} \cup J_{1}$, and this is a contraction by Remark 3.2(iii).

3.6 Lemma. If $\varphi \in \mathscr{C}(I, I), h(\varphi)=0$, and $J \subset I$ is a closed interval disjoint with $\Omega(\varphi)$ then there is $a \delta>0$ such that for any $n \geq 1$ and any $x \in J$, $\left|\varphi^{n}(x)-x\right|>\delta$.

Proof. Assume there are points $x_{k} \in J$ and integers $n(k)>0$ such that $\lim x_{k}=x_{0}$ and $\lim \left|\varphi^{n(k)}\left(x_{k}\right)-x_{k}\right|=0$ for $k \rightarrow \infty$. Let $\delta>0$. Fix a $k$ with $\left|x_{k}-x_{0}\right|<\delta / 2$ and $\left|\varphi^{n(k)}\left(x_{k}\right)-x_{k}\right|<\delta / 2$, and set $U=\left(x_{0}-\delta, x_{0}+\delta\right)$. Then $\varphi^{n(k)}(U) \cap U \neq \varnothing$, i.e., $x_{0} \in \Omega(\varphi)$ and this is impossible since clearly $x_{0} \in J$.

Before stating the next lemmas, some terminology and notation is needed. For $\varphi \in \mathscr{C}(I, I)$ and $\delta>0$, a $\delta$-chain is any finite or infinite sequence $\left\{x_{i}\right\}$ of points from $I$ with $\left|\varphi\left(x_{i}\right)-x_{i+1}\right|<\delta$ for every $i$. We say that $x$ can be chained to $y$, if for any $\delta>0$ there is a finite $\delta$-chain with the first term $x$ and the last term $y$.

Define $\nu_{0}(\varphi)$ as the minimal number with the property that for any $\varepsilon>\nu_{0}(\varphi)$ there is a $\delta>0$ such that for every $\delta$-chain $\left\{x_{i}\right\}_{i=1}^{\infty}, \lim \sup _{i \rightarrow \infty}\left|x_{i+1}-x_{i}\right|<\varepsilon$. Moreover, let $M(\varphi)$ be the set of $x \in I$, for which there is some $y \in I$ with

$$
\varphi(y) \leq x<y \leq \varphi(x) \text { or } \varphi(x) \leq y<x \leq \varphi(y)
$$

and such that $\varphi(x)$ can be chained to $y$ and $\varphi(y)$ to $x$.

3.7 Lemma. Let $\varphi \in \mathscr{C}(I, I)$. Then $\nu_{0}(\varphi)=\sup \{|\varphi(x)-x| ; \quad x \in M(\varphi)\}$.

Proof. The " $\geq$ " part is trivial. To prove the converse inequality, we may assume $\nu_{0}(\varphi)>0$. By the definition of $\nu_{0}(\varphi)$, for any $k=1,2, \ldots$ there is a $1 / k$-chain $\left\{x_{i}^{k}\right\}_{i=1}^{\infty}$ with

$$
\left|x_{i+1}^{k}-x_{i}^{k}\right|<\nu_{0}(\varphi)+1 / k \quad \text { for any } i
$$

and such that $\limsup _{i \rightarrow \infty}\left|x_{i+1}^{k}-x_{i}^{k}\right|>\nu_{0}(\varphi)-1 / k$. Let $\left(u^{k}, v^{k}\right) \in I^{2}$ be a limit point of the set $\left\{\left(x_{i}^{k}, x_{i+1}^{k}\right) ;\left|x_{i+1}^{k}-x_{i}^{k}\right|>\nu_{0}(\varphi)-1 / k\right\}$, and let $(u, v)$ be a limit point of $\left\{\left(u^{k}, v^{k}\right)\right\}_{k=1}^{\infty}$. Then clearly

$$
\begin{gathered}
\varphi(u)=v, \\
|u-v|=\nu_{0}(\varphi) .
\end{gathered}
$$

Hence to finish the proof it suffices to show that

$$
u \in M(\varphi) \text {. }
$$

Assume for simplicity that, e.g., $u<v$. For any $\delta>0$ choose $k=k(\delta)$ and $s=s(\delta)$ such that $1 / k<\delta$, and $\left|u^{k}-u\right|<\delta,\left|v^{k}-v\right|<\delta,\left|x_{s}^{k}-u^{k}\right|<\delta$, $\left|x_{s+1}^{k}-v^{k}\right|<\delta$. If $\delta$ is taken to be less than $(v-u) / 4$, then we have $x_{s+1}^{k} \geq$ $u+2 \delta$, and so there is the maximal $m=m(\delta) \geq s+1$ with $x_{j}^{k} \geq u+2 \delta$ for 
$j=s+1, \ldots, m$. Put $z(\delta)=x_{m}^{k}$, and let $z$ be a limit point of $z(\delta)$ for $\delta \rightarrow 0$. We have $z \geq u$ and by the maximality of $m, \varphi(z) \leq u$. By (3.4), $z-\varphi(z) \leq \nu_{0}(\varphi)$, hence (3.6) gives $z \leq v$. Thus $\varphi(z) \leq u<z \leq \varphi(u)$. Clearly $\varphi(z)$ can be chained to $u$ and $\varphi(u)$ to $z$, and (3.7) follows.

For $\varphi \in \mathscr{C}(I, I)$ denote $\nu(\varphi)=\inf _{n} \nu_{0}\left(\varphi^{n}\right)$.

3.8 Lemma. Let $\varphi \in \mathscr{C}(I, I)$ with $h(\varphi)=0$, and let Theorem 2.2(ii) be satisfied for $f$ replaced by $\varphi$. Then $\nu(\varphi)=0$.

Proof. Assume $\nu(\varphi)>0$. Then by Lemma 3.7, for any $n=2^{k}$ there is some $x_{n} \in M\left(\varphi^{n}\right)$ such that

$$
\left|\varphi^{n}\left(x_{n}\right)-x_{n}\right|>\nu(\varphi) / 2 .
$$

We may clearly assume that there is an increasing sequence $\{n(i)\}_{i=1}^{\infty}$ of powers of 2 with $x_{n(i)}<\varphi^{n(i)}\left(x_{n(i)}\right), i=1,2, \ldots$ (for the converse inequality the proof is similar). By the definition of $M\left(\varphi^{n(i)}\right)$, there is $y_{n(i)}$ such that $\varphi^{n(i)}\left(y_{n(i)}\right) \leq$ $x_{n(i)}<y_{n(i)} \leq \varphi^{(n(i)}\left(x_{n(i)}\right)$, so $\varphi^{n(i)}$ has a fixed point $p_{n(i)} \in\left(x_{n(i)}, y_{n(i)}\right)$, for every $i$. We may clearly assume that $\left\{p_{n(i)}\right\}_{i=1}^{\infty}$ converges monotonically to some $u$ (if not, take a subsequence). Consider the following two cases.

Case a. $p_{n(i)} \searrow u$ for $i \rightarrow \infty$. Since by Lemmas 3.4 and 3.5,

$$
p_{n(i)} \notin\left(x_{n(i+1)}, \varphi^{n(i+1)}\left(x_{n(i+1)}\right)\right)
$$

we have

$$
p_{n(i+1)}<y_{n(i+1)}<\varphi^{n(i+1)}\left(x_{n(i+1)}\right)<p_{n(i)}
$$

hence $\varphi^{n(i)}\left(x_{n(i)}\right) \searrow u=\lim p_{n(i)}$. In view of (3.8), $u \in\left(x_{n(i)}, \varphi^{n(i)}\left(x_{n(i)}\right)\right)$ for every large $i$, hence by Lemmas 3.4 and 3.5, $u \notin \operatorname{Per}(\varphi)$. Thus $u \in$ $\overline{\operatorname{Per}(\varphi)} \backslash \operatorname{Per}(\varphi)$ and by Proposition 3.1(i) there is some $v$ such that $\omega_{\varphi}(v)$ is infinite and contains $u$. By Theorem 2.2(ii) there is a periodic interval $U$ of period $2^{k}$ and such that $u \in U$ and the length of $U$ is less than $\nu(\varphi) / 2$.

If $u$ is the right-hand end-point of $U$, then by (3.8), $U \subset\left(x_{n(i)}, u\right) \subset$ $\left(x_{n(i)}, \varphi^{n(i)}\left(x_{n(i)}\right)\right)$ for every large $i$. But $U$ contains a periodic point of period $2^{k}$, i.e., a fixed point of every such $\varphi^{n(i)}$, contrary to Lemmas 3.4 and 3.5 .

So assume $U$ contains a right-hand neighborhood of $u$. Then by (3.10), for large $i,\left(y_{n(i)}, \varphi^{n(i)}\left(x_{n(i)}\right)\right) \subset U$, and $U$ is invariant with respect to $\varphi^{n(i)}$ hence we get $\left(\varphi^{n(i)}\left(y_{n(i)}\right), \varphi^{n(i)}\left(x_{n(i)}\right)\right) \subset U$, contrary to (3.8) since the length of $U$ is less than $\nu(\varphi) / 2$.

Case b. Let $p_{n(i)} \nearrow x$. Then (3.9) implies $p_{n(i)}<\varphi^{n(i+1)}\left(y_{n(i+1)}\right)<x_{n(i+1)}<$ $p_{n(i+1)}$ and similarly as in the preceding case we obtain a periodic interval $U$ of the length less than $\nu(\varphi) / 2$, with $u \in U$, and consequently, a contradiction. 


\section{Proof of the main Result}

4.1 Proposition. Let $f \in \mathscr{C}(I, I)$ have zero topological entropy. Then the following conditions are equivalent:

(i) $\operatorname{Per}(f)$ is nowhere dense;

(ii) $\operatorname{Per}(f)$ is a first category set;

(iii) $\operatorname{Per}(f)$ contains no interval.

Proof. (ii) $\Rightarrow($ i) : Assume $\operatorname{Per}(f)$ is dense in an interval $J$. Choose periodic points $p, q \in J, p<q$, and let their periods be less than $m=2^{k}$. Then $f^{m}([p, q])=[p, q]$. Indeed, by Lemma 3.4, for any $r \in[p, q] \cap \operatorname{Per}(f)$ we have $f^{m}(r) \in[p, q]$, and the rest follows from the density of $\operatorname{Per}(f)=\operatorname{Per}\left(f^{m}\right)$ in $[p, q]$, since $f^{m}$ is continuous.

Thus the restriction $g$ of $f^{m}$ to $[p, q]$ is a map $[p, q] \rightarrow[p, q]$ with $h(g)=$ 0 and $\operatorname{Per}(g)$ dense in the domain. By [1], $g$ is a homeomorphism which has no periodic points of periods other than 1 or 2. This means that $\operatorname{Per}(g)$ is a closed set and consequently, $\operatorname{Per}(g)=[p, q]=\operatorname{Per}(f) \cap[p, q]$, i.e., $\operatorname{Per}(f)$ is a second category set.

The implications (iii) $\Rightarrow$ (ii) and (i) $\Rightarrow$ (iii) are trivial.

4.2 Proposition. If $f \in \mathscr{C}(I, I)$ with $h(f)=0, \nu(f)=0$ and $\operatorname{Per}(f)$ nowhere dense, then $f$ is nonchaotic and stable.

Proof. Let $\varepsilon>0$. Since $\operatorname{Per}(f)$ is nowhere dense and $h(f)=0$ by Proposition 3.1(ii) there are closed intervals $J_{1}, \ldots, J_{k}$ such that $\Omega(f) \cap \bigcup J_{i}=\varnothing$ and

$$
\begin{aligned}
& \text { the length of } U \text { is less than } \varepsilon / 3 \text { for any connected component } \\
& U \text { of } I \backslash \bigcup J_{i} \text {. }
\end{aligned}
$$

Let $\varepsilon_{1}$ be the minimum of the lengths of $J_{i}$. By Lemma 3.6 there is $\delta_{1}>0$ such that

$$
\left|f^{n}(x)-x\right|>\delta_{1} \quad \text { for } x \in \bigcup J_{i} .
$$

Let $\varepsilon_{2}=\min \left\{\varepsilon_{1}, \varepsilon / 3\right\}$. Choose $n=2^{m}$ such that $\nu_{0}\left(f^{n}\right)<\varepsilon_{2}$, and let $\delta_{2}>0$ be such that $\sup \left|x_{i+1}-x_{i}\right|<\varepsilon_{2}$ for every $\delta_{2}$-chain generated by $f^{n}$. Let $\delta_{0}=\min \left\{\delta_{1}, \delta_{2}\right\}$. Since the operator of creating the $n$th iterate is continuous in $\mathscr{C}(I, I)$, for every $g \in \mathscr{C}(I, I)$ sufficiently near $f$ we have

$$
\left\|f^{n}-g^{n}\right\|<\delta_{0} .
$$

Fix such a $g$, and let $x \in I$. Then $\left\{g^{j n}(x)\right\}_{j=1}^{\infty}$ is clearly a $\delta_{2}$-chain generated by $f^{n}$. Hence $\left|g^{j n}(x)-g^{(j+1) n}(x)\right|<\varepsilon_{2}$ for every $j$. Let $\liminf _{j \rightarrow \infty} g^{j n}(x)$ $=\alpha, \lim \sup _{j \rightarrow \infty} g^{j n}(x)=\beta$. To finish the proof it suffices to show that $\beta-\alpha<\varepsilon$ (since the remaining inequalities can be obtained when $x$ is replaced by $\left.g^{i}(x), i=1,2, \ldots, n-1\right)$. Assume the contrary. Then by (4.1) there is a closed interval $J \subset(\alpha, \beta) \cap \bigcup J_{i}$ of the length larger than $\varepsilon_{2}$. From (4.2) and (4.3) we have $\left|y-g^{n}(y)\right|>0$ for every $y \in J$. If e.g. $g^{n}(y)<y$ for $y \in J$, 
then there is some $j$ with $g^{n j}(x)<g^{(j+1) n}(x)$ and $J \subset\left(g^{j n}(x), g^{(j+1) n}(x)\right)$. Thus $\left|g^{(j+1) n}(x)-g^{j n}(x)\right|>\varepsilon_{2}$-a contradiction.

4.3 Proposition. Let $f \in \mathscr{C}(I, I)$. If $\operatorname{Per}(f)$ contains an interval then $f$ is not stable.

Proof. Let $\operatorname{Per}(f)$ contain an interval $J$. Since $\operatorname{Per}(f)=\bigcup_{n=1}^{\infty} \operatorname{Fix}\left(f^{n}\right)$ where Fix $(f)$ is the set of fixed points of $f$, and since every Fix $\left(f^{n}\right)$ is closed, there is the smallest $m \geq 1$ such that $\operatorname{Fix}\left(f^{m}\right) \backslash \bigcup_{n<m} \operatorname{Fix}\left(f^{n}\right)$ contains a closed interval $K=[a, b] \subset J$. Clearly $K$ is periodic since it consists only of periodic points of period $m$. Since $f^{m}(x)=x$ for every $x \in K$, and since $f$ is continuous, $f$ must be strictly monotonic on $K$, i.e., $K$ is a homterval of $f$. Fix some $\delta>0$. Let $g \in \mathscr{C}(I, I)$ be defined by $g(x)=f(x)$ for $x \notin(a, b)$. For $x \in(a, b)$, let $g$ be such a perturbation of $f$ that $\|f-g\|<\delta$ and $g^{m}$ is topologically transitive in $K$ (i.e., $g^{m}$ has a trajectory dense in $K$; a similar construction, for $m=1$, is given in [15]). Then for any disjoint closed intervals $K_{a}, K_{b} \subset K$, containing $a$ and $b$, respectively, and for some $k$, we have $g^{k m}\left(K_{a}\right) \cap g^{k m}\left(K_{b}\right) \supset K_{a} \cup K_{b}$. Since $K_{a}, K_{b}$ can be choosen arbitrarily small, by Proposition $3.3 g$ is $\varepsilon$-chaotic with $\varepsilon=(b-a) / 3$, no matter how small $\delta$ is.

4.4 Proposition. Let $f \in \mathscr{C}(I, I)$. The condition (ii) of Theorem 2.2 is necessary for the stability of $f$.

Proof. Assume that condition (ii) is not satisfied. We may clearly assume that $h(f)=0$ since otherwise, by Remark 3.2 and Proposition 3.3, $f$ is chaotic and hence nonstable. Keeping the notation from Theorem 2.2, set $A=\bigcap_{n=1}^{\infty}\left(\bigcup \mathscr{I}_{n}\right)$. If int $A=\varnothing$ then $A$ is the $\omega$-limit set of $x$. Indeed, let $O(y)$ be a neighborhood of some $y \in A$. Since $\bigcup \mathscr{I}_{n} \supset \bigcup \mathscr{I}_{n+1}$, for any sufficiently large $n$ there is an index $i(n)$ such that $y \in I_{n}^{i(n)} \subset O(y)$. Moreover, since $\omega_{f}(x)$ is clearly infinite and $\omega_{f}(x) \subset \cup \mathscr{I}_{n}$, there is some $k(n)$ with $f^{k(n)}(x) \in \cup \mathscr{I}_{n}$, and hence by the periodicity of $\mathscr{I}_{n}, f^{s(n)}(x) \in I_{n}^{i(n)}$ for some $s(n)$. Taking $n \rightarrow \infty$ we get $y \in \omega_{f}(x)$.

Thus we have proved that int $A \neq \varnothing$. Let $J$ be a maximal closed interval contained in $A$. For $i \neq j$ we have $f^{i}(J) \cap f^{j}(J)=\varnothing$ since $f^{i}(J), f^{j}(J)$ belong to different components of $\bigcup \mathscr{F}_{n}$ for any sufficiently large $n$ (e.g., for $n>i+j)$. Let $\varepsilon=\operatorname{diam}(J)$ and let $\delta>0$. Choose $i, n$ such that

$$
\operatorname{diam}\left(I_{n}^{i}\right)<\delta \text { and } \operatorname{diam}\left(f\left(I_{n}^{i}\right)\right)<\delta .
$$

Let $J \subset I_{n}^{j}$ and let $m>0$ be such that $f^{m}\left(I_{n}^{j}\right)=I_{n}^{i}$. Now we choose closed intervals $J_{0}, J_{1} \subset I_{n}^{j}$ such that $\operatorname{dist}\left\{J_{0}, J_{1}\right\}>\varepsilon / 2$ and $U_{0}=f^{m}\left(J_{0}\right), U_{1}=$ $f^{m}\left(J_{1}\right)$ are intervals (nondegenerate). Such a choice is clearly possible if $f^{m}$ is nonconstant near the end-points of $I_{n}^{j}$. Otherwise we replace $f$ by a little perturbed map $\tilde{f}$ changing $f$ suitably on the intervals $I_{n}^{j}, f\left(I_{n}^{j}\right), \ldots, f^{m}\left(I_{n}^{j}\right)$ in such a way that $\|f-\tilde{f}\|<\delta$. 
Now define $g \in \mathscr{C}(I, I)$ by $g(x)=f(x)$ for $x \notin I_{n}^{i}$ and let $g\left(U_{0}\right)=$ $g\left(U_{1}\right)=g\left(I_{n}^{i}\right)=f\left(I_{n}^{i}\right)$. From (4.4), $\|f-g\|<\delta$. It is easy to see that, for $r=$ $2^{n}$, we have $g^{r}\left(J_{0}\right)=g^{r-m}\left(U_{0}\right)=I_{n}^{j} \supset J_{0} \cup J_{1}$ and similarly $g^{r}\left(J_{1}\right) \supset J_{0} \cup J_{1}$. By Proposition 3.3, $g$ is $\tilde{\varepsilon}$-chaotic for $\tilde{\varepsilon}=\varepsilon / 3=(\operatorname{diam} J) / 3$, no matter how small $\delta$ is.

4.5 Proof of Theorem 2.2. Let the assumptions be satisfied. Then $f$ is stable by Lemma 3.8, Propositions 4.1 and 4.2. If condition (i) is not satisfied then by Propositions 4.1 and 4.3, $f$ is not stable. The necessity of (ii) follows from Proposition 4.4 .

\section{REFERENCES}

1. M. Barge and J. Martin, Dense periodicity on the interval, Proc. Amer. Math. Soc. 94 (1985), 731-735.

2. L. Block, Simple periodic orbits of mappings of the interval, Trans. Amer. Math. Soc. 254 (1979), 391-398.

3. H. Chu and X. Jincheng, A counterexample in dynamical systems of the interval, Proc. Amer. Math. Soc. 97 (1986), 361-366.

4. P. Collet and J.-P. Eckmann, Iterated maps on the interval as dynamical systems, Birkhäuser, Boston, Mass., 1980.

5. W. de Melo and S. van Strien, A structure theorem in one dimensional dynamics, Delft University of Technology, 1986, preprint.

6. J. Harrison, Wandering intervals, Dynamical Systems and Turbulence, (Warwick, 1980), Lecture Notes in Math., vol. 898, Springer Verlag, Berlin, Heidelberg and New York, 1981, pp. 154-163.

7. K. Janková and J. Smital, A characterization of chaos, Bull. Austral. Math. Soc. 34 (1986), 283-292.

8. T. Y. Li and J. A. Yorke, Period three implies chaos, Amer. Math. Monthly 82 (1975), 985-992.

9. M. Misiurewicz and J. Smital, Smooth chaotic maps with zero topological entropy, Ergodic Theory Dynamical Systems 8 (1988), 421-424.

10. Z. Nitecki, Maps of the interval with closed periodic set, Proc. Amer. Math. Soc. 85 (1982), 451-456.

11. A. N. Sarkovskii, Nonwandering points and center of a continuous mapping of the line into itself, Dopovīdī Akad. Nauk Ukraïn RSR Ser. A (1964), no. 7, 865-868. (Ukrainian)

12. $\ldots$, On cycles and the structure of continuous mappings, Ukrain. Mat. Zh. 17 (1965), 104111. (Russian)

13. $\_$, On a theorem of G. D. Birkhoff, Dopovīdī Akad. Nauk Ukraïn RSR Ser. A (1967), no. 5, 429-432. (Ukrainian)

14. J. Smital, Chaotic functions with zero topological entropy, Trans. Amer. Math. Soc. 297 (1986), 269-282.

15. J. Smital and K. Smitalová, Structural stability of nonchaotic difference equations, J. Math. Anal. Appl. 90 (1982), 1-11; Errata 101 (1984), p. 324.

16. M. B. Verejkina and A. N. Sarkovskii, Recurrence in one-dimensional dynamical systems, Approximative and Qualitative Methods of the Theory of Differential-Functional Equations, Inst. Math. Akad. Nauk USSR, Kiev, 1983, pp. 35-46. (Russian)

Department of Mathematics, Charles University, Sokolovská 83, 18600 Praha, CZECHOSLOVAKIA

Department of Mathematics, Comenius University, 84215 Bratislava, CzechosloVAKIA 